\title{
Compact Toeplitz Operators for Weighted Bergman Spaces on Bounded Symmetric Domains
}

\author{
Hassan Issa
}

\begin{abstract}
Let $\Omega \subset \mathbb{C}^{d}$ be an irreducible bounded symmetric domain of type $(r, a, b)$ in its Harish-Chandra realization. We study Toeplitz operators $T_{g}^{\nu}$ with symbol $g$ acting on the standard weighted Bergman space $H_{\nu}^{2}$ over $\Omega$ with weight $\nu$. Under some conditions on the weights $\nu$ and $\nu_{0}$ we show that there exists $C\left(\nu, \nu_{0}\right)>0$, such that the Berezin transform $\tilde{g}_{\nu_{0}}$ of $g$ with respect to $H_{\nu_{0}}^{2}$ satisfies:
\end{abstract}

$$
\left\|\tilde{g}_{\nu_{0}}\right\|_{\infty} \leq C\left(\nu, \nu_{0}\right)\left\|T_{g}^{\nu}\right\|,
$$

for all $g$ in a suitable class of symbols containing $L^{\infty}(\Omega)$. As a consequence we apply a result in Engliš (Integr Equ Oper theory 33:426$455,1999)$, to prove that the compactness of $T_{g}^{\nu}$ is independent of the weight $\nu$, whenever $g \in L^{\infty}(\Omega)$ and $\nu>C$ where $C$ is a constant depending on $(r, a, b)$.

Mathematics Subject Classification (2010). 32A36, 32M15, 53C35.

Keywords. Berezin transform, Reproducing kernel, Jordan triple determinant.

\section{Introduction}

Let $\Omega \subset \mathbb{C}^{d}$ be an irreducible bounded symmetric domain of multiplicities $a$ and $b$, rank $r$ and genus $p$. For each $\nu>p-1$, we denote by $\mu_{\nu}$ the normalized standard measure on $\Omega$ with weight $\nu$, and we write $\langle\cdot, \cdot\rangle_{\nu}$ for the usual inner product on $L_{\nu}^{2}:=L^{2}\left(\Omega, d \mu_{\nu}\right)$. The weighted Bergman space $H_{\nu}^{2}:=H_{\nu}^{2}\left(\Omega, d \mu_{\nu}\right)$ is defined to be the space of all holomorphic functions in $L_{\nu}^{2}$. It is well known, that $H_{\nu}^{2}$ is closed in $L_{\nu}^{2}$ and forms a reproducing kernel Hilbert space. Via the orthogonal projection $P_{\nu}$ from $L_{\nu}^{2}$ onto $H_{\nu}^{2}$ and for a measurable symbol $f$, the Toeplitz operator $T_{f}^{\nu}$ is defined on a suitable domain in $H_{\nu}^{2}$ as the product $T_{f}^{\nu}:=P_{\nu} M_{f}$ where $M_{f}$ is the multiplication

H. Issa has been partially supported by an "Emmy-Noether scholarship" of DFG (Deutsche Forschungsgemeinschaft). 
by $f$. Moreover, for a suitable function $g: \Omega \longrightarrow \mathbb{C}$ the weighted Berezin transform $\widetilde{g}_{\nu}$ is the real analytic map on $\Omega$ given by $\widetilde{g}_{\nu}(z)=\left\langle T_{g}^{\nu} k_{\nu,[z]}, k_{\nu,[z]}\right\rangle_{\nu}$ where $k_{\nu,[z]}$ denotes the normalized Bergman kernel.

In the analysis of Toeplitz operators the Berezin transform is an important tool related to various results in operator theory (cf. $[2-5,7,8,14,15]$ ). For example, it was shown in [8] that under some condition on the weight $\nu$ the Toeplitz operator $T_{g}^{\nu}$ with bounded symbol $g$ is compact on $H_{\nu}^{2}$ if and only if $\widetilde{g}_{\nu} \in C_{0}(\Omega)$. In case of the unit disc $\Omega=\mathbb{D}$ in the complex plane, this result was generalized from bounded symbols to symbols of bounded mean oscillation $\mathrm{BMO}^{1}(\mathbb{D})$ (cf. [15]). The corresponding compactness characterization also holds true for weighted Bergman spaces over the n-dimensional unit ball $\Omega=\mathbb{B}^{n}$ and $g \in \mathrm{BMO}^{1}\left(\mathbb{B}^{n}\right)$ (cf. [3]).

Theorems of the above type have been obtained for unbounded domains, as well. In case of the Segal-Bargmann space $H^{2}\left(\mathbb{C}^{n}, d \mu_{t}\right)$ of all entire functions square integrable with respect to a $t$-dependent family of Gaussian measures $\mu_{t}$, (here $t>0$ plays the role of the time parameter in the heat flow), it was proved in [3] that for symbols $f \in \mathrm{BMO}^{1}\left(\mathbb{C}^{n}\right)$ the Toeplitz operator $T_{f}^{t}$ is bounded (respectively compact) if and only if the heat transform $\widetilde{f}^{\left(\frac{t}{2}\right)}$ at time $\frac{t}{2}$ is bounded (respectively vanishing at infinity) (cf. [3, 7]).

A natural question which arises in the study of Toeplitz operators with a fixed symbol acting on a family of weighted Bergman spaces is whether their compactness is independent of the weight parameter. By essentially using the previously mentioned results it was shown in [3] that independence in fact holds in the case of the Segal-Bargmann space $H^{2}\left(\mathbb{C}^{n}, d \mu_{t}\right)$ and the weighted Bergman spaces $H_{\nu}^{2}$ over the unit ball $\mathbb{B}^{n}$ under the assumption that $g \in \mathrm{BMO}^{1}\left(\mathbb{C}^{n}\right)$ and $g \in \mathrm{BMO}^{1}\left(\mathbb{B}^{n}\right)$, respectively. However, there are counter examples for general symbols (cf. Section 6 in [3]). As an application it follows that for functions $g \in \mathrm{BMO}^{1}\left(\mathbb{C}^{n}\right)$ the heat transform $\widetilde{g}^{(t)} \in C\left(\mathbb{C}^{n}\right)$ of $g$ vanishes at infinity for a certain time $t_{0}>0$ if and only if $\widetilde{g}^{(t)}$ vanishes at infinity for each time $t>0$. This, roughly speaking, gives some information on the heat flow "backwards in time".

In the case of a bounded symmetric domain, the weight parameter $\nu$ replaces the time parameter $t$ in the Segal-Bargmann space construction and the Berezin transform $\widetilde{g}_{\nu}$ replaces the heat transform. The aim of this paper is to prove that the compactness of $T_{g}^{\nu}$ is uniform with respect to the weight $\nu$, whenever $g \in L^{\infty}(\Omega)$ and $\nu>C$ where $C$ is a constant depending on $(r, a, b)$.

The first attempt to solve such a problem was given in [3]. One important ingredient was to obtain an estimation between the sup-norm of the heat transform of the symbol and the norm of the Toeplitz operator (cf. Theorem 11 [5], Proposition 1 and Theorem 10 in [3]) and to use the above mentioned result in [15]. In this paper, we employ a similar technique to investigate the case of a general bounded symmetric domain and we use a compactness characterization in [8] which holds for bounded symbols. We point out that under the assumption of boundedness our result is a generalization to that in [3] since we deal with a wider class of domains. However, we are not able to generalize the statement to functions of bounded mean oscillation. This 
is due to the fact that the equivalence between the vanishing of the Berezin transform at the boundary of $\Omega$ and the compactness of Toeplitz operators is not clear for such a space of symbols.

Here we state two of our main results:

Theorem A. Let $\nu>p-1, \nu_{0}>d$ with $\left|\nu-\nu_{0}\right|>\frac{r-1}{2} a$ and write $\tilde{\nu}=$ $\min \left\{\nu, \nu_{0}\right\}$. Then there exists $C\left(\nu, \nu_{0}\right)>0$ such that for all $g \in \tau_{\nu}(\Omega) \cap$ $L^{1}\left(\Omega, d \mu_{\tilde{\nu}}\right)(c f .(6))$ we have

$$
\left\|\widetilde{g}_{\nu_{0}}\right\|_{\infty} \leq C\left(\nu, \nu_{0}\right)\left\|T_{g}^{\nu}\right\| .
$$

Theorem B. Let $\Omega \subset \mathbb{C}^{d}$ be an irreducible bounded symmetric domain and suppose that

$\nu, \nu_{0}>\max \left\{d, p-1+\frac{r-1}{4} a+\sqrt{\left(\frac{r-1}{4} a\right)^{2}+\frac{r-1}{2} a\left(\frac{r-1}{2} a+p-1\right)}\right\}$.

Then for any $g \in L^{\infty}(\Omega)$ we have the equivalence:

$$
T_{g}^{\nu_{0}} \text { is compact on } H_{\nu_{0}}^{2} \text { if and only if } T_{g}^{\nu} \text { is compact on } H_{\nu}^{2} \text {. }
$$

The paper is organized as follows: In Sect. 2 we set up notations and present some of the standard results concerning irreducible bounded symmetric domains. In Sect. 3 we prove the inequality in Theorem A by a technique similar to that in [5]. An essential idea is to construct for each pair $\left(\nu, \nu_{0}\right)$ of weights a certain trace class operator on $H_{\nu}^{2}$ and represent the Berezin transform of a function as an operator trace. Section 4 is devoted to the proof of Theorem B, where we use a result in [8]. Finally, we present some open problems which are motivated by our results.

\section{Preliminaries}

Let $\Omega \subset \mathbb{C}^{d}$ be a bounded symmetric domain in its (Harish-Chandra) realization with multiplicities $a$ and $b$ and with rank $r$. In particular, $\Omega$ contains the origin and it is invariant under the natural $\mathbb{S}^{1}$-action (circular). The triple $(r, a, b)$ is called the type of $\Omega$ and it determines the domain up to biholomorphic equivalence (cf. $[6,11])$. E. Cartan proved that there exist only six types of irreducible bounded symmetric domains, the so called four classical domains and two exceptional domains of dimensions 16 and 27, respectively (cf. [6]). Moreover, the genus $p$ of $\Omega$ and the complex dimension $d$ are given by:

$$
p=2+(r-1) a+b ; \quad d:=\operatorname{dim} \Omega=r+\frac{\operatorname{ar}(r-1)}{2}+r b .
$$

The group $A u t(\Omega)$ of all biholomorphic automorphisms on $\Omega$ acts transitively on $\Omega$. Moreover, if $G$ is the connected component of $\operatorname{Aut}(\Omega)$ containing the identity (with respect to the topology of uniform convergence on compact subsets of $\Omega$ ), then $G$ is a semi-simple Lie group. Let $K$ denote the isotropy subgroup of $G$ fixing the origin. Then $K$ is a maximal compact subgroup of $G$ and $G / K \cong \Omega$ (cf. [10,12]). 
To each such $\Omega$ there is attached a unique function $h(z, w)\left(z, w \in \mathbb{C}^{d}\right)$ which is a polynomial in $z$ and $\bar{w}$, called the Jordan triple determinant and satisfies the following properties (cf. $[11,13])$ :

1. $h(z, 0)=1$ and $h(z, w)=\overline{h(w, z)}, \quad \forall z, w \in \mathbb{C}^{d}$.

2. $h(z, z)>0, \quad \forall z \in \Omega$ and $h(z, z)=0, \quad \forall z \in \partial \Omega$.

Thus for any $\lambda \in \mathbb{R}$, we fix a branch of $h(z, w)^{\lambda}$ for $z, w \in \Omega$. It is well known that for any $\nu \in \mathbb{R}(\mathrm{cf}$. [13]):

$$
\int_{\Omega} h(z, z)^{\nu-p} d v(z)<\infty \Longleftrightarrow \nu>p-1,
$$

where $d v(z)$ is the Euclidean measure on $\Omega$. In this case, we consider the normalized weighted measure:

$$
d \mu_{\nu}=c_{\nu} h(z, z)^{\nu-p} d v .
$$

The weighted Bergman space $H_{\nu}^{2}$ is the space of holomorphic functions in $L_{\nu}^{2}$. We write $K_{\nu}: \Omega \times \Omega \longrightarrow \mathbb{C}$ for the reproducing kernel of $H_{\nu}^{2}$, i.e. for any $f \in H_{\nu}^{2}, w \in \Omega$ we have:

$$
f(w)=\left[P_{\nu} f\right](w)=\left\langle f, K_{\nu}(\cdot, w)\right\rangle_{\nu}=\left\langle f, K_{\nu,[w]}\right\rangle_{\nu},
$$

where $P_{\nu}$ denotes the projection of $L_{\nu}^{2}$ onto $H_{\nu}^{2}$ and $K_{\nu,[w]}:=K_{\nu}(\cdot, w)$. The kernel $K_{\nu}(z, w)$ is related to the Jordan triple determinant $h(z, w)$ via (cf. [9]):

$$
K_{\nu}(z, w)=h(z, w)^{-\nu}, \quad \forall \nu>p-1 .
$$

For a measurable symbol $g: \Omega \longrightarrow \mathbb{C}$ the Toeplitz operator $T_{g}^{\nu}$ is given by:

$$
T_{g}^{\nu}: D\left(T_{g}^{\nu}\right):=\left\{h \in H_{\nu}^{2} \mid g h \in L_{\nu}^{2}\right\} \subset H_{\nu}^{2} \longrightarrow H_{\nu}^{2}: h \mapsto P_{\nu}(g h) .
$$

The Berezin transform $\widetilde{T}$ of an operator $T$ on $H_{\nu}^{2}$ with the domain of $T$ containing all the normalized kernels $k_{\nu,[z]}:=K_{\nu,[z]} /\left\|K_{\nu,[z]}\right\|_{\nu}$ where $z$ runs through $\Omega$, is the complex valued map defined on $\Omega$ by:

$$
\widetilde{T}(z)=\left\langle T k_{\nu,[z]}, k_{\nu,[z]}\right\rangle_{\nu}, \quad z \in \Omega \text {. }
$$

In the rest of the paper we will frequently use the symbol space:

$$
\tau_{\nu}(\Omega):=\left\{g: \Omega \longrightarrow \mathbb{C} \mid g K_{\nu,[a]} \in L^{2}\left(\Omega, d \mu_{\nu}\right) \quad \text { for all } a \in \Omega\right\} .
$$

Note that for $g \in \tau_{v}(\Omega)$ the operator $T_{g}^{\nu}$ is densely defined on $H_{\nu}^{2}$ and its Berezin transform is defined to be:

$$
\widetilde{g}_{\nu}:=\widetilde{T_{g}^{\nu}}
$$

Let $P$ be the space of all holomorphic polynomials on $\mathbb{C}^{d}$. For $f, g \in P$ we define $\left.g^{*}(z):=\overline{g(\bar{z}}\right)$ and $\partial_{f}:=f\left(\frac{\partial}{\partial z}\right)$. Let $d v(z)$ denote the Lebesgue volume measure, and equip $P$ with the Fischer inner product:

$$
\langle f, g\rangle_{F}:=\partial_{f}\left(g^{*}\right)(0)=\pi^{-d} \int_{\mathbb{C}^{d}} f(z) \overline{g(z)} e^{-|z|^{2}} d v(z) .
$$


Under the action of $K$ on $P$ which is given by: $\pi(k) f:=f \circ k$ (each $k \in K$ can be extended to a linear map on $\mathbb{C}^{d}(\mathrm{cf}$. [12])), $P$ admits a Peter Weyl decomposition:

$$
P=\oplus P_{m},
$$

where $m=\left(m_{1}, \ldots, m_{r}\right) \in \mathbb{N}_{0}^{r}$ with $m_{1} \geq \cdots \geq m_{r} \geq 0$ (in all what follows the multi-indices $m$ will always have this ordering). Note that each $P_{m}$ is a subspace of $P_{|m|}$, the space all homogeneous polynomials on $\mathbb{C}^{d}$ of degree $|m|$ (cf. [9]).

The importance of this decomposition is given by the following theorem:

Theorem 1. [1] The spaces $P_{m}$ are K-invariant irreducible and orthogonal under $\langle\cdot, \cdot\rangle_{F}$. Moreover, if $H$ is a Hilbert space of analytic functions on $\Omega$ with $K$-invariant inner product $\langle\cdot, \cdot \cdot\rangle$, then $P_{m}$ is orthogonal to $P_{n}$ under $\langle\cdot, \cdot\rangle$ whenever $m \neq n$. Moreover, $\langle\cdot, \cdot\rangle\rangle$ is proportional to $\langle\cdot, \cdot\rangle_{F}$ on each $P_{m}$.

In particular, this holds for the space $H_{\nu}^{2}$ and the constant of proportionality was calculated in [9]:

$$
\langle f, g\rangle_{F}=(\nu)_{m}\langle f, g\rangle_{\nu},
$$

where $(\nu)_{m}$ is the generalized Pochhammer symbol:

$$
(\nu)_{m}:=\prod_{j=1}^{r} \frac{\Gamma\left(m_{j}+\nu-\frac{j-1}{2} a\right)}{\Gamma\left(\nu-\frac{j-1}{2} a\right)} .
$$

Since each $P_{m}$ is closed in $P$ ( $P_{m}$ is finite dimensional), it admits a reproducing kernel $K^{m}(z, w)$, i.e.

$$
f(w)=\left\langle f, K^{m}(\cdot, w)\right\rangle_{F}, \quad \forall f \in P_{m} .
$$

The relation between these reproducing kernels and the Bergman kernel was given in [9]:

Theorem 2. [9] For all $\nu \in \mathbb{C}$ and all $z, w \in \Omega$, we have:

$$
h(z, w)^{-\nu}=\sum_{m_{1} \geq \cdots \geq m_{r} \geq 0}(\nu)_{m} K^{m}(z, w) .
$$

The series converges uniformly and absolutely on compact subsets of $\Omega \times \bar{\Omega}$.

Finally, we relate the orthonormal basis of the Fischer norm to the orthonormal basis of $H_{\nu}^{2}$ :

Proposition 1. Let $\nu>p-1, d_{m}=\operatorname{dim} P_{m}$, and $\left\{\psi_{j}^{m}\right\}_{j=1, \ldots, d_{m}}$ be an orthonormal basis of $\left(P_{m},\langle\cdot, \cdot\rangle_{F}\right)$. Then

$$
\mathcal{B}:=\left\{e_{j}^{m}:=(\nu)_{m}^{\frac{1}{2}} \psi_{j}^{m} \mid m \text { and } j=1, \ldots, d_{m}\right\}
$$

is an orthonormal basis of $\left(H_{\nu}^{2},\langle\cdot, \cdot\rangle_{\nu}\right)$. Here $m$ runs through the previously mentioned ordering. 
Proof. Let $j \neq k$ then $\left\langle\psi_{j}^{m}, \psi_{k}^{m}\right\rangle_{\nu}=\frac{1}{(\nu)_{m}}\left\langle\psi_{j}^{m}, \psi_{k}^{m}\right\rangle_{F}=0$. Moreover, for $m \neq n$ (cf. Theorem 1)

$$
\left\langle\psi_{j}^{m}, \psi_{i}^{n}\right\rangle_{\nu}=0 ; \quad \forall j \leq d_{m}, i \leq d_{n} .
$$

Also, note that:

$$
\left\langle(\nu)_{m}^{\frac{1}{2}} \psi_{j}^{m},(\nu)_{m}^{\frac{1}{2}} \psi_{j}^{m}\right\rangle_{\nu}=(\nu)_{m}\left\langle\psi_{j}^{m}, \psi_{j}^{m}\right\rangle_{\nu}=\left\langle\psi_{j}^{m}, \psi_{j}^{m}\right\rangle_{F}=1 .
$$

Now, since the polynomials are dense in $H_{\nu}^{2}$ it follows that the functions in $\mathcal{B}$ form a complete orthonormal system of $H_{\nu}^{2}$.

\section{Upper Estimation of the Berezin Transform}

In this section we prove Theorem A using a similar technique to that in [5]. Under the assumptions on the weights $\nu$ and $\nu_{0}$ and for each $z \in \Omega$, we find a trace class operator $T_{z}^{\nu, \nu_{0}}$ on $H_{\nu}^{2}$ where $\left\|T_{z}^{\nu, \nu_{0}}\right\|_{t r}$ is independent of $z$ and one has $\operatorname{tr}\left(T_{g}^{\nu} T_{z}^{\nu, \nu_{0}}\right)=\frac{c_{\nu}}{c_{\nu_{0}}} \widetilde{g}_{\nu_{0}}(z)$. We then apply a standard estimate for the trace norm to obtain the inequality (1).

For $X \in \mathcal{L}\left(H_{\nu}^{2}\right)$ define the function $K_{\nu}^{X}(z, w):=\left(X^{*} K_{\nu}(\cdot, w)\right)(z)$ on $\Omega \times \Omega$. By slightly modifying the arguments in [5], one can prove the following (cf. [5] corollary to Theorem 6):

Proposition 2. Let $g \in \tau_{\nu}(\Omega)$ and $X \in \mathcal{L}\left(H_{\nu}^{2}\right)$ with $\nu>p-1$. Suppose the following conditions hold:

1. $T_{g}^{\nu} \in \mathcal{L}\left(H_{\nu}^{2}\right)$.

2. $T_{g}^{\nu} X$ is of trace class on $H_{\nu}^{2}$.

3. $\int_{\Omega} \int_{\Omega}|g(z)|\left|K_{\nu}(w, z)\right|\left|K_{\nu}^{X}(w, z)\right| d \mu_{\nu}(z) d \mu_{\nu}(w)<\infty$.

Then

$$
\operatorname{tr}\left(T_{g}^{\nu} X\right)=\int_{\Omega} g(z) \overline{K_{\nu}^{X}(z, z)} d \mu_{\nu}(z) .
$$

The basic idea in the proof of Theorem A is to construct a trace class operator $X=T_{0}^{\nu, \nu_{0}}$ on $H_{\nu}^{2}$ satisfying $K_{\nu}^{X}(z, w)=h(z, w)^{\nu_{0}-\nu}$. In order to do that, we fix $\left\{\psi_{j}^{m}\right\}_{j=1, \ldots, d_{m}}$ as an orthonormal basis of $\left(P_{m},\langle\cdot, \cdot\rangle_{F}\right)$. Then, the reproducing kernel $K^{m}(z, w)$ of $P_{m}$ is given by:

$$
K^{m}(z, w)=\sum_{j=1}^{d_{m}} \psi_{j}^{m}(z) \overline{\psi_{j}^{m}(w)} .
$$

Now, for each $m \in \mathbb{N}_{0}^{r}$ such that $m_{1} \geq \cdots \geq m_{r} \geq 0$ and each $j=1, \ldots, d_{m}$, we denote by $P_{j}^{m}$ the orthogonal projection from $H_{\nu}^{2}$ to the one-dimensional space spanned by $e_{j}^{m}$ :

$$
P_{j}^{m} f:=\left\langle f, e_{j}^{m}\right\rangle_{\nu} e_{j}^{m} \quad f \in H_{\nu}^{2} .
$$

Hence:

$$
\begin{aligned}
\overline{K_{\nu}^{P_{j}^{m}}(z, w)}=\left[P_{j}^{m} K_{\nu}(\cdot, z)\right](w) & =\left\langle K_{\nu}(\cdot, z), e_{j}^{m}\right\rangle_{\nu} e_{j}^{m}(w) \\
& =(\nu)_{m} \overline{\psi_{j}^{m}(z)} \psi_{j}^{m}(w) .
\end{aligned}
$$


Now, define the operator $T_{0}^{\nu, \nu_{0}}$ on $H_{\nu}^{2}$ as the infinite sum:

$$
T_{0}^{\nu, \nu_{0}}:=\sum_{m_{1} \geq \cdots \geq m_{r} \geq 0} \frac{\left(\nu-\nu_{0}\right)_{m}}{(\nu)_{m}} \sum_{j=1}^{d_{m}} P_{j}^{m} .
$$

Theorem 3. For $\nu>p-1$ and $\nu_{0}>d$ the operator $T_{0}^{\nu, \nu_{0}}$ is of trace class on $H_{\nu}^{2}$ and

$$
K_{\nu}^{T_{0}^{\nu, \nu_{0}}}(z, w)=h(z, w)^{\nu_{0}-\nu} .
$$

Proof. For each $l \in \mathbb{N}$, consider the operator

$$
S_{l}^{\nu, \nu_{0}}:=\sum_{m_{1} \geq \cdots \geq m_{r} \geq 0,|m| \leq l} \frac{\left(\nu-\nu_{0}\right)_{m}}{(\nu)_{m}} \sum_{j=1}^{d_{m}} P_{j}^{m} .
$$

We write:

$$
\frac{\left(\nu-\nu_{0}\right)_{m}}{(\nu)_{m}}=\prod_{j=1}^{r} \frac{\Gamma\left(m_{j}+\nu-\nu_{0}-\frac{j-1}{2} a\right)}{\Gamma\left(\nu-\nu_{0}-\frac{j-1}{2} a\right)} \frac{\Gamma\left(\nu-\frac{j-1}{2} a\right)}{\Gamma\left(m_{j}+\nu-\frac{j-1}{2} a\right)} .
$$

Therefore, by Stirling's and as $m_{j} \longrightarrow \infty$ we have:

$$
\frac{\Gamma\left(m_{j}+\nu-\nu_{0}-\frac{j-1}{2} a\right)}{\Gamma\left(m_{j}+\nu-\frac{j-1}{2} a\right)} \sim m_{j}^{-\nu_{0}} .
$$

Since $\nu_{0}>p-1 \geq 1$, it follows that the operators $S_{l}^{\nu, \nu_{0}}$ converge to $T_{0}^{\nu, \nu_{0}}$ in the norm of $\mathcal{L}\left(H_{\nu}^{2}\right)$. Moreover, by an application of the Cauchy-Schwarz Theorem we know that:

$$
K_{\nu}^{S_{l}^{\nu, \nu_{0}}}(z, w) \longrightarrow K_{\nu}^{T_{0}^{\nu, \nu_{0}}}(z, w),
$$

as $l \longrightarrow \infty$ uniformly on compact subsets of $\Omega \times \Omega$ (cf. Proposition 3-(7) in [5]). Together with Theorem 2 and the equalities (7), (10), and (11) we obtain:

$$
\begin{aligned}
K_{\nu}^{T_{0}^{\nu, \nu_{0}}}(z, w) & =\sum_{m_{1} \geq \cdots \geq m_{r} \geq 0} \frac{\left(\nu-\nu_{0}\right)_{m}}{(\nu)_{m}} \sum_{j=1}^{d_{m}} K_{\nu}^{P_{j}^{m}}(z, w) \\
& =\sum_{m_{1} \geq \cdots \geq m_{r} \geq 0} \frac{\left(\nu-\nu_{0}\right)_{m}}{(\nu)_{m}} \sum_{j=1}^{d_{m}}(\nu)_{m} \psi_{j}^{m}(z) \overline{\psi_{j}^{m}(w)} \\
& =\sum_{m_{1} \geq \cdots \geq m_{r} \geq 0}\left(\nu-\nu_{0}\right)_{m} K^{m}(z, w) \\
& =h(z, w)^{\nu_{0}-\nu} .
\end{aligned}
$$

In order to prove that $T_{0}^{\nu, \nu_{0}}$ is of trace class, note that $\left\|\sum_{j=1}^{d_{m}} P_{j}^{m}\right\|_{\mathrm{tr}}=d_{m}=$ $\operatorname{dim} P_{m}$. One has the inclusion $P_{m} \subset P_{|m|}$ and $P_{|m|}$ admits $\left\{c_{\alpha} z^{\alpha}|| \alpha \mid=\right.$ $\left.|m|, \alpha \in \mathbb{N}_{0}^{d}\right\} \quad\left(c_{\alpha}\right.$ are normalized constants) as an orthonormal basis with respect to the Fischer inner product. Hence it follows that

$$
d_{m} \leq \frac{(|m|+d-1) !}{|m| !(d-1) !} .
$$


Therefore,

$$
\begin{aligned}
\left\|T_{0}^{\nu, \nu_{0}}\right\|_{t r} & =\left|\sum_{m_{1} \geq \cdots \geq m_{r} \geq 0} d_{m} \prod_{j=1}^{r} \frac{\Gamma\left(m_{j}+\nu-\nu_{0}-\frac{j-1}{2} a\right)}{\Gamma\left(\nu-\nu_{0}-\frac{j-1}{2} a\right)} \frac{\Gamma\left(\nu-\frac{j-1}{2} a\right)}{\Gamma\left(m_{j}+\nu-\frac{j-1}{2} a\right)}\right| \\
& \leq \sum_{m \in \mathbb{N}^{r}} \frac{(|m|+d-1) !}{|m| !(d-1) !} \prod_{j=1}^{r}\left|\frac{\Gamma\left(m_{j}+\nu-\nu_{0}-\frac{j-1}{2} a\right)}{\Gamma\left(\nu-\nu_{0}-\frac{j-1}{2} a\right)} \frac{\Gamma\left(\nu-\frac{j-1}{2} a\right)}{\Gamma\left(m_{j}+\nu-\frac{j-1}{2} a\right)}\right| .
\end{aligned}
$$

Now, as $|m| \rightarrow \infty$, and by Stirling's formula again we have:

$$
\begin{aligned}
\frac{(|m|+d-1) !}{|m| !(d-1) !} \sim \frac{|m|^{d-1}}{(d-1) !} & =\frac{1}{(d-1) !}\left\{\sum_{j=1}^{r} m_{j}\right\}^{d-1} \\
& =\sum_{|\gamma|=d-1} \frac{1}{\gamma !} m^{\gamma} \leq c \prod_{i=1}^{r}\left(1+m_{i}\right)^{d-1}
\end{aligned}
$$

where $c>0$ is a suitable constant independent of $m$. Therefore, as $m_{j} \longrightarrow \infty$ it follows that:

$$
\begin{aligned}
& \prod_{j=1}^{r} \frac{\Gamma\left(m_{j}+\nu-\nu_{0}-\frac{j-1}{2} a\right)}{\Gamma\left(m_{j}+\nu-\frac{j-1}{2} a\right)} \frac{(|m|+d-1) !}{(|m|) !(d-1) !} \\
& \quad \leqslant c \prod_{j=1}^{r} \frac{\Gamma\left(m_{j}+\nu-\nu_{0}-\frac{j-1}{2} a\right)}{\Gamma\left(m_{j}+\nu-\frac{j-1}{2} a\right)}\left(1+m_{j}\right)^{d-1} \sim c \prod_{j=1}^{r}\left(1+m_{j}\right)^{-\nu_{0}+d-1} .
\end{aligned}
$$

Since $\nu_{0}>d$ this shows that $T_{0}^{\nu, \nu_{0}}$ is of trace class.

We want to apply Proposition 2 to $X=T_{0}^{\nu, \nu_{0}}$ and in order to check condition (3) therein, we need the generalized Forelli-Rudin inequalities which can be found in $[8,9]$ :

Lemma 1. Consider the integral

$$
J_{\lambda, \gamma}(z)=\int_{\Omega}|h(z, w)|^{-(\lambda+\gamma)} h(w, w)^{\lambda-p} d v(w),
$$

where $z \in \Omega, \gamma \in \mathbb{R}$ and $\lambda>p-1$. Let

$$
\mathbb{A}_{\lambda, \gamma}=\left\{\alpha \in \mathbb{R} \mid \exists C>0 \text { s.t. }\left|J_{\lambda, \gamma}(z)\right| \leq C h(z, z)^{-\alpha}, \quad \text { for all } z \in \Omega\right\},
$$

then:

1. $\mathbb{A}_{\lambda, \gamma}=[\gamma, \infty)$, if $\gamma>\frac{r-1}{2} a$.

2. $\mathbb{A}_{\lambda, \gamma}=[0, \infty)$, if $\gamma<-\frac{r-1}{2} a$.

According to Theorem 3 and to the above lemma, we are able now to establish the following theorem:

Theorem 4. Let $\nu>p-1, \nu_{0}>d$ with $\left|\nu-\nu_{0}\right|>\frac{r-1}{2} a$, and write $\tilde{\nu}=\min \left\{\nu, \nu_{0}\right\}$. Moreover, suppose that $g \in \tau_{\nu}(\Omega) \cap L^{1}\left(\Omega, d \mu_{\tilde{\nu}}\right)$ and that $T_{g}^{\nu}$ is bounded. Then

$$
\operatorname{tr}\left(T_{g}^{\nu} T_{0}^{\nu, \nu_{0}}\right)=\frac{c_{\nu}}{c_{\nu_{0}}} \widetilde{g}_{\nu_{0}}(0)
$$


Proof. The first step is to verify condition (3) of Proposition 2 i.e. to prove the convergence of the following integral

$$
\int_{\Omega} \int_{\Omega}\left|g(z) \| K_{\nu}(w, z)\right|\left|K_{\nu}^{T_{0}^{\nu, \nu_{0}}}(w, z)\right| d \mu_{\nu}(z) d \mu_{\nu}(w) .
$$

Using (4) and (12) we obtain:

$$
\begin{aligned}
& \int_{\Omega} \int_{\Omega}|g(z)|\left|K_{\nu}(w, z)\right|\left|K_{\nu}^{T_{0}^{\nu, \nu_{0}}}(w, z)\right| d \mu_{\nu}(z) d \mu_{\nu}(w) \\
& \quad=c_{\nu, \nu_{0}} \int_{\Omega} \int_{\Omega}|h(w, z)|^{-\left(\nu+\left(\nu-\nu_{0}\right)\right)} h(w, w)^{\nu-p} d v(w)|g(z)| h(z, z)^{\nu-p} d v(z) .
\end{aligned}
$$

Now, we apply Lemma 1 for $\lambda=\nu$ and $\gamma=\nu-\nu_{0}$. Since $|\gamma|>\frac{r-1}{2} a$, we have two case:

(a) In case $\nu>\nu_{0}$, Lemma 1 (1) implies that $\nu-\nu_{0} \in \mathbb{A}_{\lambda, \gamma}$, which means that:

$$
\int_{\Omega}|h(w, z)|^{-\left(\nu+\left(\nu-\nu_{0}\right)\right)} h(w, w)^{\nu-p} d v(w) \leq C h(z, z)^{\nu_{0}-\nu} .
$$

So, we have to check if

$$
\int_{\Omega} h(z, z)^{\nu_{0}-\nu} h(z, z)^{\nu-p}|g(z)| d v(z)=\frac{1}{c_{\nu_{0}}} \int_{\Omega}|g(z)| d \mu_{\nu_{0}}(z)<\infty .
$$

This is true since $g \in L^{1}\left(\Omega, d \mu_{\nu_{0}}\right)$.

(b) In case of $\nu_{0}>\nu$, we have $\gamma<-\frac{r-1}{2} a$. By Lemma 1 (2), we know that $0 \in \mathbb{A}_{\lambda, \gamma}$ i.e.

$$
\int_{\Omega}|h(w, z)|^{-\left(\nu+\left(\nu-\nu_{0}\right)\right)} h(w, w)^{\nu-p} d v(w) \leq C .
$$

Therefore, we have to investigate

$$
\int_{\Omega}|g(z)| h(z, z)^{\nu-p} d v(z)<\infty .
$$

But this is also true since in this case we have $g \in L^{1}\left(\Omega, d \mu_{\nu}\right)$.

Hence, we can apply Proposition 2 with $X=T_{0}^{\nu, \nu_{0}}$ to obtain:

$$
\begin{aligned}
& \operatorname{tr}\left(T_{g}^{\nu} T_{0}^{\nu, \nu_{0}}\right)=\int_{\Omega} g(z) \overline{K_{\nu}^{T_{0}^{\nu, \nu}}(z, z)} d \mu_{\nu}(z) \\
& =c_{\nu} \int_{\Omega} g(z) h(z, z)^{\nu_{0}-\nu} h(z, z)^{\nu-p} d v(z) \\
& =\frac{c_{\nu}}{c_{\nu_{0}}} \int_{\Omega} g(z) d \mu_{\nu_{0}}(z)=\frac{c_{\nu}}{c_{\nu_{0}}} \tilde{g}_{\nu_{0}}(0) .
\end{aligned}
$$


Our aim now is to extend the above Theorem from $z=0$ to an arbitrary $z \in \Omega$. More precisely, under the assumptions of Theorem 4 and for each $z \in \Omega$ we find a trace class operator $T_{z}^{\nu, \nu_{0}}$ such that $\operatorname{tr}\left(T_{g}^{\nu} T_{z}^{\nu, \nu_{0}}\right)=\frac{c_{\nu}}{c_{\nu_{0}}} \widetilde{g}_{\nu_{0}}(z)$ and the trace norm $\left\|T_{z}^{\nu, \nu_{0}}\right\|_{t r}=\left\|T_{0}^{\nu, \nu_{0}}\right\|_{t r}$ is independent of $z \in \Omega$.

For each $z \in \Omega$ we consider the automorphism $\phi_{z}$ which interchanges $z$ and zero. We define the linear operator $U_{\nu, z}$ on $H_{\nu}^{2}$ by

$$
U_{\nu, z} f:=\left(f \circ \phi_{z}\right) \cdot J \phi_{z}{ }^{\frac{\nu}{p}}, \quad \forall f \in H_{\nu}^{2},
$$

where $J \phi_{z}$ denotes the complex Jacobian of $\phi_{z}$.

Using the transformation formula of the unweighted Bergman kernel, one can prove:

Proposition 3. For all $z \in \Omega$, the operator $U_{\nu, z}$ is self-adjoint and unitary i.e.

$$
U_{\nu, z}^{-1}=U_{\nu, z}^{*}=U_{\nu, z}
$$

For each $S \in \mathcal{L}\left(H_{\nu}^{2}\right)$, and each $z \in \Omega$ we define $S_{\nu, z} \in \mathcal{L}\left(H_{\nu}^{2}\right)$ by:

$$
S_{\nu, z}:=U_{\nu, z} S U_{\nu, z}
$$

The following lemma is found in ([8], Lemma 4):

Lemma 2. For all $a, z \in \Omega$, the Berezin transform $\widetilde{S_{\nu, a}}$ of $S_{\nu, a}$ is given by:

$$
\widetilde{S_{\nu, a}}(z)=\widetilde{S}\left(\phi_{a}(z)\right) \text {. }
$$

One can easily check a slightly more general version of Lemma 6 in [8]:

Lemma 3. Let $\nu>p-1$ then for any $g \in \tau_{\nu}(\Omega)$ and any $a \in \Omega$ we have:

$$
U_{\nu, a} T_{g}^{\nu} U_{\nu, a}=T_{g \circ \phi_{a}}^{\nu},
$$

where both sides are interpreted as operators with domain $D\left(T_{g \circ \phi_{a}}^{\nu}\right)(c f .(5))$.

According to (14), we know that for any $z \in \Omega$ the operator

$$
T_{z}^{\nu, \nu_{0}}:=U_{\nu, z} T_{0}^{\nu, \nu_{0}} U_{\nu, z}
$$

is of trace class and satisfies $\left\|T_{z}^{\nu, \nu_{0}}\right\|_{t r}=\left\|T_{0}^{\nu, \nu_{0}}\right\|_{t r}$. Moreover, according to Theorem 4 together with (15) and (16) we obtain:

Corollary 1. Let $\nu>p-1$ and $\nu_{0}>d$ with $\left|\nu-\nu_{0}\right|>\frac{r-1}{2} a$. Moreover, suppose that $g \in \tau_{\nu}(\Omega) \cap L^{1}\left(\Omega, d \mu_{\tilde{\nu}}\right)$, where $\tilde{\nu}=\min \left\{\nu, \nu_{0}\right\}$, and that $T_{g}^{\nu}$ is bounded. Then we have:

$$
\operatorname{tr}\left(T_{g}^{\nu} T_{z}^{\nu, \nu_{0}}\right)=\frac{c_{\nu}}{c_{\nu_{0}}} \widetilde{g}_{\nu_{0}}(z) \quad \forall z \in \Omega .
$$

Proof. We apply Theorem 4:

$$
\begin{aligned}
\operatorname{tr}\left(T_{g}^{\nu} T_{z}^{\nu, \nu_{0}}\right) & =\operatorname{tr}\left(T_{g}^{\nu} U_{\nu, z} T_{0}^{\nu, \nu_{0}} U_{\nu, z}\right)=\operatorname{tr}\left(U_{\nu, z} T_{g}^{\nu} U_{\nu, z} T_{0}^{\nu, \nu_{0}}\right) \\
& =\operatorname{tr}\left(T_{g \circ \phi_{z}}^{\nu} T_{0}^{\nu, \nu_{0}}\right)=\frac{c_{\nu}}{c_{\nu_{0}}}\left(\widehat{g \circ \phi_{z}}\right)_{\nu_{0}}(0) \\
& =\frac{c_{\nu}}{c_{\nu_{0}}} \widetilde{g}_{\nu_{0}}\left(\phi_{z}(0)\right)=\frac{c_{\nu}}{c_{\nu_{0}}} \widetilde{g}_{\nu_{0}}(z) .
\end{aligned}
$$


Proof of Theorem A: Let $T_{g}^{\nu}$ be bounded, then by Corollary 1 and by a standard estimate for the trace norm we have for all $z \in \Omega$ :

$$
\begin{aligned}
\left|\tilde{g}_{\nu_{0}}(z)\right| & \leq C_{1}\left(\nu, \nu_{0}\right)\left|\operatorname{tr}\left(T_{g}^{\nu} T_{z}^{\nu, \nu_{0}}\right)\right| \\
& \leq C_{1}\left(\nu, \nu_{0}\right)\left\|T_{g}^{\nu}\right\|\left\|T_{z}^{\nu, \nu_{0}}\right\|_{t r} \\
& =C_{1}\left(\nu, \nu_{0}\right)\left\|T_{g}^{\nu}\right\|\left\|T_{0}^{\nu, \nu_{0}}\right\|_{t r} \\
& \leq C\left(\nu, \nu_{0}\right)\left\|T_{g}^{\nu}\right\|,
\end{aligned}
$$

where $C_{1}\left(\nu, \nu_{0}\right)$ and $C\left(\nu, \nu_{0}\right)$ are suitable constants independent of $g$ and $z$. Therefore:

$$
\left\|\tilde{g}_{\nu_{0}}\right\|_{\infty} \leq C\left(\nu, \nu_{0}\right)\left\|T_{g}^{\nu}\right\| .
$$

Clearly, the trace norm $\left\|T_{0}^{\nu, \nu_{0}}\right\|_{t r}$ also depends on $r$ and $a$. However, we omit indicating this fact in the constant $C\left(\nu, \nu_{0}\right)$.

\section{Compactness Criteria}

In the following, we establish the equivalence stated in Theorem B. For this, we need a density result for a class of Toeplitz operators together with Theorem A and the main result in [8]. Similar to the corresponding result in [5] on Toeplitz operators acting on the Segal-Bargmann space, and by purely functional analytic methods one can prove the following:

Theorem 5. Let $\nu>p-1$, then

$$
\left\{T_{g}^{\nu} \quad \mid \text { g continuous with compact support in } \Omega\right\}
$$

is norm dense in the space of all compact operators acting on $H^{2}\left(\Omega, d \mu_{\nu}\right)$.

In order to prove Theorem B, we use the equivalence between the compactness of the Toeplitz operator and the vanishing of the Berezin transform at the boundary of a bounded symmetric domain (cf. Theorem A in [8]):

Theorem 6. [8] Let

$$
\nu>p-1+\frac{r-1}{4} a+\sqrt{\left(\frac{r-1}{4} a\right)^{2}+\frac{r-1}{2} a\left(\frac{r-1}{2} a+p-1\right)} .
$$

Denote by $C_{0}(\Omega)$ the space of functions vanishing on $\partial \Omega$. Then for any $g \in L^{\infty}(\Omega)$ the following two conditions are equivalent:

1. $T_{g}^{\nu}$ is compact on $H_{\nu}^{2}$.

2. $\widetilde{g}_{\nu} \in C_{0}(\Omega)$.

In fact, the above theorem is generalized to all finite sums of Toeplitz operators with bounded symbols and other equivalent conditions can be given. In our proof, the conditions on the weights $\nu$ and $\nu_{0}$ in Theorem B is 
owned to an application of Theorem 6. The key of proving Theorem B is the following:

Theorem 7. Let $\Omega \subset \mathbb{C}^{d}$ be an irreducible bounded symmetric domain and suppose that

$$
\nu, \nu_{0}>\max \left\{d, p-1+\frac{r-1}{4} a+\sqrt{\left(\frac{r-1}{4} a\right)^{2}+\frac{r-1}{2} a\left(\frac{r-1}{2} a+p-1\right)}\right\} .
$$

Then for any $g \in L^{\infty}(\Omega)$ the following are equivalent:

(1) $\widetilde{g}_{\nu_{0}} \in C_{0}(\Omega)$.

(2) $\widetilde{g}_{\nu} \in C_{0}(\Omega)$, for all $\nu$ such that $\left|\nu-\nu_{0}\right|>\frac{r-1}{2} a$.

(3) $T_{g}^{\nu_{0}}$ is compact on $H_{\nu_{0}}^{2}$.

(4) $T_{g}^{\nu}$ is compact on $H_{\nu}^{2}$, for all $\nu$ such that $\left|\nu-\nu_{0}\right|>\frac{r-1}{2} a$.

(5) $\widetilde{g}_{\nu} \in C_{0}(\Omega)$, for some $\nu$ such that $\left|\nu-\nu_{0}\right|>\frac{r-1}{2} a$.

(6) $T_{g}^{\nu}$ is compact on $H_{\nu}^{2}$, for some $\nu$ such that $\left|\nu-\nu_{0}\right|>\frac{r-1}{2} a$.

Proof. (i) We prove the implication $(6 \Longrightarrow 1)$ : Suppose that $T_{g}^{\nu}$ is compact for some $\nu$ such that $\left|\nu-\nu_{0}\right|>\frac{r-1}{2} a$. Then by Theorem A there exists $C\left(\nu, \nu_{0}\right)>0$ independent of $g$ such that:

$$
\left\|\widetilde{g}_{\nu_{0}}\right\|_{\infty} \leq C\left(\nu, \nu_{0}\right)\left\|T_{g}^{\nu}\right\|
$$

Since $T_{g}^{\nu}$ is compact, Theorem 5 tells us that there is a sequence $\left(g_{k}\right)_{k \in \mathbb{N}}$ of continuous functions with compact support such that:

$$
\left\|T_{g_{k}}^{\nu}-T_{g}^{\nu}\right\| \stackrel{k \rightarrow \infty}{\longrightarrow} 0
$$

which shows that

$$
\left\|\widetilde{g}_{\nu_{0}}-\widetilde{\left(g_{k}\right)} \nu_{\nu_{0}}\right\|_{\infty} \stackrel{k \rightarrow \infty}{\longrightarrow} 0 .
$$

But each $\widetilde{\left(g_{k}\right)} \nu_{\nu_{0}} \in C_{0}(\Omega)$, hence $\widetilde{g}_{\nu_{0}} \in C_{0}(\Omega)$.

(ii) By the same argument it is easy to show the implication $(3 \Longrightarrow 2)$ using the inequality $\left\|\tilde{g}_{\nu}\right\|_{\infty} \leq C\left(\nu_{0}, \nu\right)\left\|T_{g}^{\nu_{0}}\right\|_{\nu}$.

Finally, we remark that by Theorem 6 we have $(1 \Longleftrightarrow 3),(2 \Longleftrightarrow 4)$, and $(5 \Longleftrightarrow 6)$. The implications $(4 \Longrightarrow 6)$ and $(2 \Longrightarrow 5)$ are trivial and we obtain the proof.

Proof of Theorem $B$ Let $\left(\nu, \nu_{0}\right)$ be two weights satisfying (20) and suppose that $T_{g}^{\nu_{0}}$ is compact. Then by the previous theorem it is sufficient to prove that $T_{g}^{\nu}$ is compact for $\left|\nu-\nu_{0}\right| \leq \frac{r-1}{2} a$. Set $\gamma:=\max \left\{\nu_{0}, \nu\right\}$ and let $\nu_{1}=\gamma+1+\frac{r-1}{2} a$. Then we have

$$
\min \left\{\nu_{1}-\nu_{0}, \nu_{1}-\nu\right\}>\frac{r-1}{2} a \Longrightarrow\left(T_{g}^{\nu_{1}} \text { is compact } \Longrightarrow T_{g}^{\nu} \text { is compact }\right) \text {. }
$$




\section{Open Problems}

Finally, we would like to collect some open problems which are motivated by our results.

Question 1: Is the obstruction $\left|\nu-\nu_{0}\right|>\frac{r-1}{2} a$ in Theorem A necessary to obtain the inequality (1)?

Question 2: In [3] it was proved that in the case of the unit ball $\Omega=\mathbb{B}^{n} \subset \mathbb{C}^{n}$ Theorem B holds for symbols $g \in \mathrm{BMO}^{1}\left(\mathbb{B}^{n}\right)$ of bounded mean oscillation. This class of symbols strictly contains the bounded measurable functions. So one may ask if it is possible to extend Theorem B to the case of $\mathrm{BMO}^{1}(\Omega)$ symbols for an arbitrary bounded symmetric domain $\Omega \subset \mathbb{C}^{n}$.

Question 3: Let $S_{p}\left(H_{\nu}^{2}\right)$ denote the Schatten-p-class over $H_{\nu}^{2}$ and assume that:

$\nu, \nu_{0}>\max \left\{d, p-1+\frac{r-1}{4} a+\sqrt{\left(\frac{r-1}{4} a\right)^{2}+\frac{r-1}{2} a\left(\frac{r-1}{2} a+p-1\right)}\right\}$.

For every $g \in L^{\infty}(\Omega)$, and all $1 \leq p<\infty$, is it true that:

$$
T_{g}^{\nu_{0}} \in S_{p}\left(H_{\nu_{0}}^{2}\right) \text { if and only if } T_{g}^{\nu} \in S_{p}\left(H_{\nu}^{2}\right) ?
$$

\section{Acknowledgments}

I would like to take this opportunity to thank Prof. Wolfram Bauer who has been most generous with his time and ideas, calling my attention to the above problems. It is my pleasure to dedicate this work to Ibn L. Hassan Al-Moaammal and to my wife Aliye.

Open Access. This article is distributed under the terms of the Creative Commons Attribution Noncommercial License which permits any noncommercial use, distribution, and reproduction in any medium, provided the original author(s) and source are credited.

\section{References}

[1] Arazy, J., Upmeier, H.: Invariant inner product in spaces of holomorphic functions on bounded symmetric domains. Doc. Math. 2, 213-261 (1997)

[2] Axler, S., Zheng, D.: Compact operators via the Berezin transform. Indiana Univ. Math. J. 47(2), 387-400 (1998)

[3] Bauer, W., Coburn, L.A., Isralowitz, J.: Heat flow, BMO, and the compactness of Toeplitz operators. J. Funct. Anal. 259, 57-78 (2010)

[4] Bauer, W., Furutani, K.: Compact operators and the pluriharmupperonic Berezin transform. Int. J. Math. 19(6), 645-669 (2008)

[5] Berger, C., Coburn, L.: Heat flow and Berezin-Toeplitz estimates. Am. J. Math. 116(3), 563-590 (1994)

[6] Cartan, E.: Sur les domaines bornés homogènes de l' espace de n-variables complexes. Abh. Math. Semin. Univ. Hamburg 11, 116-162 (1935) 
[7] Coburn, L.A., Isralowitz, J., Li, B.: Toeplitz operators with BMO symbols on the Segal-Bargmann space. Trans. Am. Math. Soc. 363, 3015-3030 (2011)

[8] Engliš, M.: Compact Toeplitz operators via the Berezin transform on bounded symmetric domains. Integr. Equ. Oper. Theory 33, 426-455 (1999)

[9] Faraut, J., Koranyi, A.: Function spaces and reproducing kernels on bounded symmetric domains. J. Funct. Anal. 88(1), 64-89 (1990)

[10] Helgason, S.: Differential Geometry, Lie Groups, and Symmetric Spaces. American Mathematical Society, Providence (2001)

[11] Loos, O.: Bounded symmetric domains and Jordan pairs. Mathematical Lectures. University of California at Irvine, Irvine (1977)

[12] Narasimhan, R.: Several Complex Variables. Chicago Lectures in Mathematics. University of Chicago Press, Chicago (1971)

[13] Upmeier, H.: Toeplitz Operators and Index Theory in Several Complex Variables. In: Operator Theory: Advances and Applications, vol. 81, Birkhäuser, Basel (1996)

[14] Zhu, K.: Operator Theory in Function Spaces. In: Mathematical Surveys and Monographs, vol. 138, 2nd edn. The American Mathematical Society, Providence (2007)

[15] Zorboska, N.: Toeplitz operators with BMO symbols and the Berezin transform. Int. J. Math. Math. Sci. 46, 2929-2945 (2003)

Hassan Issa $(\bowtie)$

Mathematisches Institut

Georg-August-Universität

Bunsen-str. 3-5

37073 Göttingen

Germany

e-mail: hissa@uni-math.gwdg.de;

baladalamin@hotmail.com

Received: December 16, 2010.

Revised: May 5, 2011. 